\title{
Diversity and abundance of comammox bacteria in the sediments of an urban lake
}

Yangfan Xu ${ }^{\text {a, b }}$, Jing Lu ${ }^{c}$, Yuchun Wang ${ }^{\text {d, e }}$, Guanglong Liu ${ }^{\text {a, b }}$, Xiaoqiong Wan ${ }^{\text {a, b }}$, Yumei Hua ${ }^{\text {a, b }}$,

Duanwei Zhu ${ }^{\text {a, b }}$, Jianwei Zhao ${ }^{\text {a, b, * }}$

Running head: Diversity and abundance of comammox bacteria

a Laboratory of Eco-Environmental Engineering Research of Huazhong Agricultural University,

Wuhan 430070, China

b Key Laboratory of Arable Land Conservation (Middle and Lower Reaches of Yangtze River),

Ministry of Agriculture, Wuhan 430070, China

c Australian Rivers Institute, Griffith University, Nathan, QLD 4111, Australia

d State Key Laboratory of Simulation and Regulation of Water Cycle in River Basin, Beijing 10038,

China.

e Department of water environment, China Institute of water resources and hydropower research,

Beijing 10038, China

*Correspondence: Jianwei Zhao

Tel.: $+86-27-85669068$

E-mail: jwzhao2@163.com

Postal address: No.1, Shizishan Street, Hongshan District, Wuhan, Hubei Province, 430070, People’s

Republic of China

\section{Abstract:}

Aims: Although comammox have been discovered in a variety of ecosystems, there are few studies in urban lakes. This paper attempted to confirm whether this microbe exists in urban lakes and to 
determine the factors influencing its existence.

Methods and Results: This study investigated the diversity and abundance of comammox bacteria in sediments of a typical urban lake in China, and the ecological relationship with other ammonia-oxidizing microorganisms. The phylogenetic analysis indicated that there was only clade A in the comammox strain in the sediment of Lake Donghu. The comammox co-existed with ammonia-oxidizing archaea (AOA), ammonia-oxidizing bacteria (AOB) and anaerobic ammonia-oxidizing (anammox) bacteria in the sediment of this lake. The abundances of the ammonia monooxygenase genes for comammox, $\mathrm{AOA}, \mathrm{AOB}$, and anammox $16 \mathrm{~S}$ rRNA were $2.43 \times 10^{8}, 1.07 \times$ $10^{8}, 3.24 \times 10^{7}$, and $3.21 \times 10^{11}$ copies $\mathrm{g}^{-1}$ dry sediment, respectively. Moreover, the amo $A$ gene abundance of comammox was positively correlated with that of AOA and AOB. The redundancy analysis (RDA) showed that the abundance of the comammox amoA gene was negatively correlated with various nitrogen related indicators in both the sediment and the water column, indicating that eutrophication may inhibit the growth of comammox.

Conclusions: Comammox bacteria play an important ecological role in the nitrogen cycle of urban lake sediments.

Significance and Impact of the Study: Our results indicated comammox bacteria were widespread in urban lakes and eutrophication may inhibit their growth.

\section{Keywords}

Comammox; Nitrospira; Diversity; Abundance; Lake Sediment

\section{Introduction}

Nitrification is a key component of the nitrogen cycle in lakes. Traditional nitrification processes had been generally considered involving two steps (Shiozaki et al. 2016), ammonia-oxidizing archaea 
(AOA) and ammonia-oxidizing bacteria (AOB) first oxidize ammonia to nitrite, then nitrite-oxidizing bacteria (NOB) oxidize nitrite to nitrate (Konneke et al. 2005). However, Costa et al. (2006) speculated from the perspective of the kinetic theory that there was probably a nitrifying microorganism that can directly oxidize ammonia to nitrate and grow in a low ammonium environment. The discovery of complete ammonia oxidizers (comammox) confirmed the presence of this microbe (Daims et al. 2015; van Kessel et al. 2015). The genome of this microbe contains both gene encoding ammonia monooxygenase, hydroxylamine dehydrogenase and nitrite oxidoreductase, indicating that they have the potential for the full-scale ammoxidation (Daims et al. 2015; van Kessel et al. 2015). This discovery has profoundly expanded our understanding of the ammonia oxidation process. Therefore, the existence and ecological effects of this oxidation process in natural ecosystems need to be further evaluated.

Although comammox was discovered just a few years ago, it has been found to co-exist with AOA, and AOB, and widely distributed in different ecosystems using molecular biology techniques, such as in drinking water treatment plants (DWTPs), atmospheric particulate matter, soil, estuarine sediments, and wastewater treatment plants (WWTPs) (Gao et al. 2016; Wang et al. 2017; Fowler et al. 2018; Wang et al. 2018; Yu et al. 2018). AOA were previously thought to dominate in environments with low ammonium concentrations, such as oceans or oligotrophic lakes (Herrmann et al. 2011; Hou et al. 2013), and AOB have a higher abundance in nutrient-rich waters and soils (Dang et al. 2010; Prosser and Nicol 2012). However, with further research on comammox community and its environments, it showed that comammox may have advantages over AOA and AOB in low ammonium environments (Kits et al. 2017), and ammonium concentrations could also affect the diversity of commomox community (Yu et al. 2018). In some drinking water influent, river, groundwater and urban waterway 
sediment samples, the proportion of comammox in the total number of ammonia-oxidizing microorganisms was higher than $70 \%$ (Xia et al. 2018). And it may be an important functional community in the nitrification process in low dissolved oxygen (DO) environments (Roots et al. 2019). The similar results were also found in the study of tidal sediments in which middle and lower sediments with a sharp drop in dissolved oxygen concentration provided better growth conditions for comammox bacteria than surface sediments (Jiang et al. 2019).

Based on the similarity of the amoA gene, comammox can be divided into two major branches, clade A and clade B (Daims et al. 2015; van Kessel et al. 2015). Many studies have shown that these two branches may have ecological differentiation in nature. Pjevac et al. (2017) designed a PCR primer to amplify the comammox amoA gene to verify the distribution of comammox in various environments (Freshwater biofilm; Forest soil; Rice paddy soil; WWTPs; DWTPs et al), and the results showed that the abundance of the two branches in varying samples were significantly different. High-throughput sequencing of river sediments revealed that the concentration of comammox clade A was higher than that of clade B (Xia et al. 2018), while comammox in the groundwater-fed rapid sand filter samples was dominated by clade B (Fowler et al. 2018). Studies in forest soils have found that the abundances of comammox clade A and AOA were comparable when the soil is alkaline, but in the acidic soil, comammox clade A is more abundant than AOA (Hu and He 2017). The rate of nitrogen deposition also has an effect on ammonia-oxidizing microorganisms. With the increased of nitrogen deposition rate, the amoA gene abundance of comammox Nitrospira clade B was significantly increased (Shi et al. 2018). When applying nutrients to the soil, the relative abundance of comammox Nitrospira clade A increases with the increasing nutrient input gradient, while clade B was the opposite. This may be due to the different physiological characteristics between the comammox Nitrospira clade (Wang et al. 
2019a).

Anaerobic ammonia-oxidizing (anammox) bacteria can also oxidize ammonia using nitrite as an electron acceptor under anaerobic or anoxic conditions (Mulder et al. 1995). Therefore, AOA and AOB can provide the growth substrate of nitrite for anammox bacteria (Zhao et al. 2019a). It has been found that $\mathrm{AOA}, \mathrm{AOB}$, and anammox bacteria can co-exist in habitats, such as soils, rivers, lake sediments, and marine sediments (Kuypers et al. 2003; Chen and Gu 2017; Wang et al. 2019b). Some studies indicated that anammox bacteria can also co-exist with comammox bacteria (Annavajhala et al. 2018), and these four types of microorganisms were both detected in the sequencing batch reactor (SBR) (Pamela et al. 2017). However, these microorganisms all require ammonium as a substrate for their reaction, and thus there may be a complex competition-collaboration relationship between them. The study using SBR to treat sludge digester liquor found that $\mathrm{AOB}, \mathrm{NOB}$, comammox bacteria and anammox bacteria could coexist, and the removal efficiency of $\mathrm{NH}_{4}{ }^{+}-\mathrm{N}$ was as high as $98 \%$ (Wu et al. 2019). The proportion of partial-nitrification, comammox, and anammox removal of $\mathrm{NH}_{4}{ }^{+}-\mathrm{N}$ in this study was $55.11 \%, 25.43 \%$, and $18.28 \%$, respectively. And the one-step nitrification process of comammox bacteria avoids the production of nitrous oxide which was often comes from the AOA and AOB-dominated ammoxidation processes (Daims et al. 2015; van Kessel et al. 2015). Therefore, comammox may play a very important role in the ecosystem nitrogen cycle.

The nitrogen content of many urban lakes in the middle and lower reaches of the Yangtze River in China is usually high (Chen et al. 2009; Wu et al. 2010; Hou et al. 2013). This high nitrogen concentrations could changed the community structure of the AOA and AOB (Yang et al. 2016a). However, the existence and diversity of comammox bacteria in these urban lakes, and its relationships with other ammonia-oxidizing microorganisms are still unclear. We hypothesized that the nutritional 
status of the water would have an effect on the growth of comammox bacteria. Therefore, we selected a typical urban lake, Lake Donghu, and investigated the comammox bacteria diversity in sediments, the correlation of comammox bacteria abundance with environmental factors, and its ecological relationships with $\mathrm{AOA}, \mathrm{AOB}$, and anammox bacteria.

\section{Materials and methods}

\section{Sample Collection}

Lake Donghu $\left(30^{\circ} 33^{\prime} \mathrm{N}, 114^{\circ} 23^{\prime} \mathrm{E}\right)$ is a shallow lake (average depth: $2.5 \mathrm{~m}$; surface area $\left.32 \mathrm{~km}^{2}\right)$ in the middle reaches of the Yangtze River, located in the urban area of Wuhan city, China. In this study, samples were collected from five sub-lakes in Lake Donghu, namely, Lake Guozheng (LGZ), Lake Tuan (LT), Lake Tangling (LTL), Lake Hou (LH), and Lake Miao (LM) (Fig. 1). The water quality of the first three sub-lakes was better than that of the last two sub-lakes (Ji et al. 2019). Sediments around the lake have been polluted by domestic sewage and non-point sources, while sediments in the center of the lake were less disturbed by humans. Thus, we collected the lake sediments of each sub-lake. At each sampling sites, the $\mathrm{pH}, \mathrm{DO}$, and water temperature $(\mathrm{T})$ of the overlying water were recorded using the YSI EXO2 Multi-Parameter Water Quality Analyzer (YSI Inc., Yellow Springs, Ohio, USA) in July 2018. At the same time, both overlying water and the surface sediment samples were collected. The surface $10 \mathrm{~cm}$ sediment samples were collected using a cylindrical sampler and stored in sterile ziplock bags. Then, these sediments were taken back to the laboratory on ice. A portion of the sediment sample was stored at $4{ }^{\circ} \mathrm{C}$ for the determination of basic physical and chemical parameters, and the rest was stored in a $-80{ }^{\circ} \mathrm{C}$ ultra-low temperature freezer for DNA extraction.

\section{Environmental Physicochemical Analyses}

The water column total phosphorus (TP), total nitrogen (TN), and chemical oxygen demand (COD) 
were measured according to the Chinese national standard methods (GB/T 11893-1989, GB/T

11894-1989, China) and national environmental protection standard method (HJ/T 399-2007, China), respectively. The concentrations of ammonia nitrogen $\left(\mathrm{NH}_{4}^{+}-\mathrm{N}\right)$, nitrite nitrogen $\left(\mathrm{NO}_{2}^{-}-\mathrm{N}\right)$, and nitrate nitrogen $\left(\mathrm{NO}_{3}{ }^{-}-\mathrm{N}\right)$ in the sediment after $\mathrm{KCl}$ extraction was measured determined according to Bao (1981). The $\mathrm{pH}$ of the sediment after $\mathrm{KCl}$ extraction was measured using a $\mathrm{pH}$ meter (METTLER TOLEDO, Switzerland). The sediment organic content was measured by the Walkley-Black chromic acid wet oxidation method (Bao 1981). The sediment TP, and TN were determined by the perchloric acid-sulfuric acid method and the Kjeldahl method (Bao 1981), respectively.

\section{DNA extraction and PCR amplification}

Approximately $0.4 \mathrm{~g}$ of the sediment sample was placed in a Lysing Matrix E tube to extract the total genomic DNA in the sediment using the Fast DNA ${ }^{\circledR}$ Spin for Soil kit (MPBIO, USA) according to the manufacturer's protocols. The purity and concentration of the extracted DNA samples were examined using a super differential spectrophotometer (NanoPhotometer-N60, IMPLEN, Germany). The extracted DNA samples were stored at $-20{ }^{\circ} \mathrm{C}$ for subsequent analysis. PCR amplification was performed in a total volume of $30 \mu \mathrm{L}$ containing: $3 \mu \mathrm{L}$ of $10 \times \operatorname{TransTaq}^{\circledR}-\mathrm{T}$ Buffer (Trans Gen, China), $2 \mu \mathrm{L}$ of dNTPs $(2.5 \mathrm{mM}$, Trans Gen, China $), 1 \mu \mathrm{L}$ of each primer $(10 \mu \mathrm{M}), 0.2 \mu \mathrm{L}$ of Trans Taq ${ }^{\circledR}-\mathrm{T}$ DNA Polymerase (Trans Gen, China), and $2 \mu \mathrm{L}$ of template DNA. Comammox gene in sediments was amplified using the specific primers comamoA AF (AGGNGAYTGGGAYTTCTGG) and comamoA

SR (CCGVACATACATRAAGCCCAT) (Wang et al. 2018). PCR amplification procedures consisted of initial denaturation at $96{ }^{\circ} \mathrm{C}$ for $5 \mathrm{~min}$, followed by 12 cycles of $96{ }^{\circ} \mathrm{C}$ for $30 \mathrm{~s}, 62{ }^{\circ} \mathrm{C}$ for $30 \mathrm{~s}, 72{ }^{\circ} \mathrm{C}$ for $30 \mathrm{~s}$, and then 37 cycles of $96^{\circ} \mathrm{C}$ for $30 \mathrm{~s}, 50{ }^{\circ} \mathrm{C}$ for $30 \mathrm{~s}, 72{ }^{\circ} \mathrm{C}$ for $30 \mathrm{~s}$, followed by a final extension at $72^{\circ} \mathrm{C}$ for $10 \mathrm{~min}$. The expected length of the PCR product was $436 \mathrm{bp}$. 


\section{Clone Library Construction and Phylogenetic Analysis}

The purified DNA was ligated with PMD18-T (TAKARA, Dalian, China), and the ligated product was transformed into Escherichia coli competent cells, then coated with X-gal, IPTG, and ampicillin-resistant LB plates. These coated plates were incubated at $37^{\circ} \mathrm{C}$ in the incubator for $16 \mathrm{~h}$. After incubation, 69 positive clones were picked out, inoculation cultured and extracted for the plasmid. Plasmids with the desired fragments were sequenced using an ABI 3730x1 DNA Sequencer (Applied Biosystems, USA). The obtained sequences were assigned to the same operational taxonomic unit (OTU) using the MOTHUR (version 1.34.1, Schloss et al. 2009) program with a maximum distance of $3 \%$. The adjacency method using MEGA 6.0 software (Tamura et al. 2013) was used for the phylogenetic tree construction, and 1000 guided analyses was repeated to estimate the confidence values of the tree nodes.

\section{Quantitative PCR}

Quantitative PCR (q-PCR) was performed on QuantStudio $^{\mathrm{TM}} 6$ Flex Real Time PCR System (Thermo Fisher Scientific, Singapore) to estimate the abundance of comammox amoA, AOA amoA, AOB amoA, and anammox 16S rRNA genes, with three replicates each. Primers used for comammox amo $A$, AOA amo $A$, AOB amo $A$, and anammox 16S rRNA genes were Ntsp-amo $A$ 359R/Ntsp-amo $A$ 162F (Fowler et al. 2018), GenAOAF/GenAOAR (Meinhardt et al. 2015), amoA-1Fmod/GenAOBR (Meinhardt et al. 2015), and AMX809F/AMX1066R (Ji et al. 2012), respectively (Table S1). The quantification of each sample was based on the fluorescent dye SYBR Green I, which bound to the double-stranded DNA during PCR amplification. The quantitative PCR reactions for comammox amoA, $\mathrm{AOA} a m o A, \mathrm{AOB}$ amoA, and anammox 16S rRNA genes were performed in a $10 \mu \mathrm{L}$ reactions mixture which included $5 \mu \mathrm{L}$ of T5 Fast qPCR Mix $(2 \times), 0.4 \mu \mathrm{L}$ of each primer $(10 \mu \mathrm{M}), 0.2 \mu \mathrm{L}$ of $\operatorname{ROX}$ 
Reference Dye II $(50 \times), 3 \mu \mathrm{L}$ of distilled deionized water $\left(\mathrm{ddH}_{2} \mathrm{O}\right)$, and $1 \mu \mathrm{L}$ of template DNA. The quantitative PCR amplification conditions for comammox amo $A$ gene was: $95^{\circ} \mathrm{C}$ for $1 \mathrm{~min}$; followed by 40 cycles of $95{ }^{\circ} \mathrm{C}$ for $10 \mathrm{~s}, 52^{\circ} \mathrm{C}$ for $20 \mathrm{~s}, 72{ }^{\circ} \mathrm{C}$ for $30 \mathrm{~s}$; AOA amo $A$ gene was: $95^{\circ} \mathrm{C}$ for $1 \mathrm{~min}$; followed by 40 cycles of $95{ }^{\circ} \mathrm{C}$ for $10 \mathrm{~s}, 53{ }^{\circ} \mathrm{C}$ for $15 \mathrm{~s}, 72{ }^{\circ} \mathrm{C}$ for $30 \mathrm{~s}$; AOB amo $A$ gene was: $95{ }^{\circ} \mathrm{C}$ for $1 \mathrm{~min}$; followed by 40 cycles of $95^{\circ} \mathrm{C}$ for $10 \mathrm{~s}, 52{ }^{\circ} \mathrm{C}$ for $15 \mathrm{~s}, 72^{\circ} \mathrm{C}$ for $30 \mathrm{~s}$; and anammox $16 \mathrm{~S}$ rRNA gene was: $95{ }^{\circ} \mathrm{C}$ for $1 \mathrm{~min}$; followed by 40 cycles of $95{ }^{\circ} \mathrm{C}$ for $10 \mathrm{~s}, 53{ }^{\circ} \mathrm{C}$ for $20 \mathrm{~s}, 72{ }^{\circ} \mathrm{C}$ for $30 \mathrm{~s}$. The genes to be amplified were separately cloned into the $\mathrm{pGEM}^{\circledR}$-T vector (Promega, Madison, WI, USA) to generate a standard plasmid, and the obtained plasmid DNA was serially diluted by 6 - 8 gradients to prepare a standard curve. Then, the qPCR standard curve was repeated three times, and the amplification efficiencies of the four genes were $83.30 \%, 91.33 \%, 91.22 \%$, and $92.59 \%$, respectively, and the correlation coefficients $\left(r^{2}\right)$ were all greater than 0.99 . A negative control containing no template DNA was performed after the same qPCR method.

\section{Statistical analyses}

One-way ANOVA and Tukey test $(P<0.05)$ were performed using SPSS 20.0 software (Hulsen $e t$ al. 2008) to determine the difference of physicochemical parameters amongst sampling locations both in sediments and the overlying water, and significant differences of microbial abundances amongst sampling locations. In the detrended correspondence analysis (DCA), the lengths of gradient in AOA, AOB, comammox bacteria, and anammox bacteria were shorter than 3.0. So, redundancy analysis (RDA) were used to analyze the relationship between the community structure of the ammoxidation microbes and environmental factors by Canoco 4.5 software (Lepš and Šmilauer 2003).

\section{Nucleotide sequence accession numbers}

The nucleotide sequences of comammox amoA obtained in this study were deposited in the 
GenBank database under accession MK948223-MK948291.

\section{Results}

\section{Physicochemical Characteristics of Samples}

The concentration of DO, TP, TN and COD in the overlying water of Lake Miao were higher than those of other four sub-lakes $(P<0.05$; Table 1.). The concentration of TP and COD in the overlying water of Lake Hou were also higher than Lake Guozheng, Lake Tuan, and Lake Tangling, and the value of $\mathrm{pH}$ and $\mathrm{NO}_{2}^{-}-\mathrm{N}$ content in the sediments of this lake were the highest $(P<0.05)$ in the five sub-lakes. Whereas, the highest content of TP and organic matter (OM) in sediments was found in Lake Guozheng $(P<0.05)$.

\section{Diversity of the Comammox amo $A$ gene}

A total of 69 comammox amoA gene sequences were obtained from the constructed cloned library.

Using Mothur software, the sequences with similarity greater than $97 \%$ were classified into one OTU, and a total of six OTUs and three clusters were obtained, namely Nitrosa Cluster, Nitrificans Cluster, and Inopinata Cluster (Fig. 2). Although the phylogenetic tree divided Nitrospira nitrosa and Nitrospira sp into two branches, they belonged to the Nitrosa Cluster. And Candidatus Nitrospira nitrosa has low homology with Nitrospira sp $(<50 \%, 1000$ replicates $)$.

All three Clusters belong to comammox clade A. Among them, OTU 1 and OTU 5 belonged to Candidatus Nitrospira nitrosa, and OTU 2 was located on the Nitrospira sp branch. A total of 48 sequences of these three OTUs accounted for $69.6 \%$ of all commmox amo $A$ gene sequences. And these sequences were highly homologous to the drinking water system, paddy soil, and tidal sediments in the database (>95\%, 1000 replicates). OTU 3 was assigned to the Nitrificans Cluster, and it shared a high degree of homology (>95\%, 1000 replicates) with Candidatus Nitrospira nitrificans and river 
sediments. Nitrificans Cluster and Inopinata Cluster contained approximately the same number of sequences, accounting for $15.9 \%$ and $14.5 \%$ of all comammox amoA gene sequences, respectively. The OTU 4 and OTU 6 in the Inopinata Cluster have higher homology to samples from drinking water plants and ocean sediments.

\section{Gene abundance of Comammox amoA, $\mathrm{AOA}$ amoA, $\mathrm{AOB}$ amoA and Anammox 16S rRNA}

The abundance of the comammox amoA gene was not significantly different amongst LGZ, LT, LTL, and LH sites (approx. $10^{8}$ copies $\mathrm{g}^{-1}$ dry sediment), but they were all significantly higher than that of the LM site ( $10^{7}$ copies $\mathrm{g}^{-1}$ dry sediment; Fig. 3). Comammox amoA gene abundance in the five sub-lakes was higher than that of $\mathrm{AOA}$ amoA gene and $\mathrm{AOB}$ amoA gene.

The average of AOA amo $A$ gene abundance was $(1.07 \pm 1.1) \times 10^{8}$ copies $\mathrm{g}^{-1}$ dry sediment, with the highest detected at the LTL site $\left(2.79 \times 10^{8}\right.$ copies $\mathrm{g}^{-1}$ dry sediment), while the lowest at the LM site $\left(2.73 \times 10^{5}\right.$ copies $\mathrm{g}^{-1}$ dry sediment). And the average of AOB amoA gene copy number was $(3.24 \pm 1.8)$ $\times 10^{7}$ copies $\mathrm{g}^{-1}$ dry sediment. There had significant difference in the AOB amoA gene abundance at these five sub-lakes. The maximum value of AOB amoA gene abundance observed at LGZ was $5.20 \times$ $10^{7}$ copies $\mathrm{g}^{-1}$ dry sediment, the lowest at the $\mathrm{LM}\left(2.42 \times 10^{6}\right.$ copies $^{-1}$ dry sediment $)$.

The average of abundance of anammox $16 \mathrm{~S}$ rRNA gene was $(3.24 \pm 0.45) \times 10^{11}$ copies $\mathrm{g}^{-1}$ dry sediment, which was much higher than that of the other three aerobic strains amoA. The gene abundance of anammox $16 \mathrm{~S}$ rRNA at LTL was up to $3.85 \times 10^{11}$ copies $\mathrm{g}^{-1}$ dry sediment, and the lowest was found at $\mathrm{LH}$.

\section{Effect of environmental variables on abundance of Comammox, AOA, AOB and Anammox}

The gene abundance of these four bacteria and environmental factors were analyzed using RDA. The first two RDA axes together explained $99.4 \%$ of the total variance for the gene abundance (Fig. 4). 
Among them, the distance between LGZ, LT and LTL sites were relatively close, indicating that there was a small difference between them and the water quality was relatively close. The abundance of comammox amoA gene was positively correlated with that of the $\mathrm{AOA}$ amoA and $\mathrm{AOB}$ amoA gene. The abundance of AOA amoA gene was positively correlated with OM and TN in sediments, while AOB amoA gene was negatively correlated with $\mathrm{NO}_{3}{ }^{-} \mathrm{N}$, TP of the sediments. Anammox $16 \mathrm{~S}$ rRNA was positively correlated with TP, TN, and OM. Comammox amoA gene was positively correlated with sediment $\mathrm{OM}$ and $\mathrm{TN}$, but negatively correlated with $\mathrm{NO}_{3}^{-}-\mathrm{N}, \mathrm{TP}$, and $\mathrm{NH}_{4}{ }^{+}-\mathrm{N}$ in sediments and overlying water indicators.

\section{Discussion}

This study investigated the diversity and abundance of the comammox in the sediments of an urban lake and effects of the water quality and sediment properties on comammox distribution.

Candidatus Nitrospira nitrosa was the dominant comammox in the sediment of Lake Donghu, followed by Candidatus Nitrospira nitrificans, and then Candidatus Nitrospira inopinata. These genus of comammox were also widely found in other ecosystems, such as wastewater treatment systems (Wang et al. 2018; Zheng et al. 2019), drinking water treatment systems (Pinto et al. 2016; Wang et al. 2017), and tidal wetlands (Yu et al. 2018). In the study of the sewage treatment plant, most of the samples were mainly composed of Candidatus Nitrospira nitrosa (Keene-Beach and Noguera 2018). N. nitrosa cluster was also found to be dominant in heavily polluted river sediments (Zhao et al. 2019b), which was similar to our study. These results indicate that $N$. nitrosa can adapt to higher substrate concentrations and may have an important role in eutrophic waters.

The comammox genus found in this study all belong to clade A, indicating comammox clade A may play an important ecological role in the nitrogen cycle of lake sediments in the middle reaches of 
the Yangtze River. Most of the current research found comammox clade A and clade B co-existed in rapid gravity sand filter, WWTPs, Vercelli rice rhizosphere, SBR, and soil (Palomo et al. 2016; Pjevac et al. 2017; Roots et al. 2019; Wang et al. 2019a). However, only comammox clade A was found in our study and in lake sediment, freshwater biofilms and some artificial ecosystems, and only comammox clade B was found in the forest soil (Pjevac et al. 2017). Comammox clade A and clade B were detected in agricultural soils samples (Zhao et al. 2019b). The presence of clade A and clade B in different habitats suggests that niche differentiation have occurred between clade A and B on horizontal habitat dimensions. Some studies found that clade B lived in an oligotrophic environment mainly because it has Amt-type transporters with higher affinity for ammonia (Palomo et al. 2018; Zhao et al. 2019b). But Clade A was more inclined to eutrophic environment because clade A has low ammonia affinity and high absorptive Rh-type transporters (Palomo et al. 2018; Zhao et al. 2019b). Transporters differences may be an important reason for their niche differentiation, and it may also be the reason that only clade A existed in the eutrophic Lake Donghu.

Many studies have shown that the abundance of AOA and AOB may be affected by the nutritional status of lakes (Yang et al. 2016b; Pollet et al. 2018). However, whether the nutritional status of the lake can affect the abundance of comammox is still unclear. In this study, the three sub-lakes of LGZ, LT and LTL were clustered into one group in the RDA analysis, which all had better water quality conditions and higher abundance of comammox (averaged at $3.11 \times 10^{8}$ copies $\mathrm{g}^{-1}$ dry sediment), compared to the sub-lakes of LM and LH. Additionally, the water quality of Lake LM was the worst, and its comammox amo $A$ gene abundance was also the lowest $\left(1.40 \times 10^{7}\right.$ copies $^{-1}$ dry sediment $)$. This indicated that the abundance of comammox amo $A$ gene seemed to be affected by the water quality of Lake Donghu, with the higher level of eutrophication reducing the abundance of comammox amo $\mathrm{A}$ 
gene. It has been demonstrated that comammox has a strong affinity for ammonia and may be more competitive at low ammonium concentrations (Daims et al. 2015; van Kessel et al. 2015). Therefore, the lower ammonium environment in the three sub-lakes of LGZ, LT and LTL in this study might have favored the growth of comammox, compared to the other two sub-lakes, which might be the reason for their higher abundance.

The comammox amoA gene abundance of the sediment in this study was $1.40 \times 10^{7} \sim 3.38 \times 10^{8}$ copies $\mathrm{g}^{-1}$ dry sediment, which were close to those filtered drinking water in the four drinking water systems (Wang et al. 2017) and biofilter in the freshwater recirculating aquaculture systems (Bartelme et al. 2017). However, it was lower than the rice paddy soil and WWTPs ( $10^{11}$ copies $\mathrm{g}^{-1}$ dry sediment $)$ (Pjevac et al. 2017). The abundance of the comammox amoA gene in fertilized soils (Wang et al. 2019a) was close to that in the river and lake sediments (Yu et al. 2018). However, it was lower than that in the acidic forest soils ( $\mathrm{Hu}$ and $\mathrm{He} 2017$ ), and the highest abundance value was found in groundwater wells (Pjevac et al. 2017). These findings suggest that the proportion of comammox in $\mathrm{N}$ bacteria community might differ in different ecosystems, thus its contribution to the nitrogen cycle may also differ in various environments. Therefore, comammox bacteria may have some selectivity to habitats in the process of ammonia conversion in ecosystems.

Comammox bacteria has been found to co-exist with $\mathrm{AOA}$ and $\mathrm{AOB}$ in various habitats such as soil, tidal flat sediments, and WWTPs (Shi et al. 2018; Jiang et al. 2019; Roots et al. 2019), and it can also co-exist with anammox bacteria (Annavajhala et al. 2018). The present study confirmed that comammox bacteria can co-exist with $\mathrm{AOA}, \mathrm{AOB}$, and anammox bacteria in lake sediments. Moreover, there was a significant positive correlation between the abundance of comammox bacteria and $\mathrm{AOA} / \mathrm{AOB}$, indicating that there might be a synergistic relationship between these bacteria. This 
synergistic relationship is likely due to the complex interaction between these bacteria from utilizing ammonia, nitrite and organic matter in the $\mathrm{N}$ cycling (Daims et al. 2015; van Kessel et al. 2015). We hypothesized that the presence of comammox bacteria might be negatively correlated with anammox bacteria, as comammox directly oxidizes ammonia to nitrate, which reduces the source of nitrite supply to anammox bacteria and may adversely affect the growth of anammox bacteria (Daims et al. 2015; van Kessel et al. 2015; Annavajhala et al. 2018). However, the correlation between comammox and anammox bacteria were not significant in the present study. This might be due to the fact that comammox bacteria use of organic matter and total nitrogen may affect the deamination of anammox bacteria. The competition-collaboration mechanism and driving factors among the four bacteria still need further research.

RDA analysis in the present study showed that the abundance of the comammox amoA gene was positively correlated with $\mathrm{OM}$ and $\mathrm{TN}$ in sediments, which was similar to the results in tidal sediments (Yu et al. 2018). Studies have shown that comammox has a high affinity for $\mathrm{NH}_{4}^{+}-\mathrm{N}$ and was suitable for growth at low $\mathrm{NH}_{4}{ }^{+}-\mathrm{N}$ concentrations (Kits et al. 2017). In this study, the $\mathrm{NH}_{4}{ }^{+}-\mathrm{N}$ concentration of the sediment has a negative correlation with comammox amoA abundance, which was similar to the findings of a study of tidal flat sediments in the Yangtze River estuary (Jiang et al. 2019). Moreover, $\mathrm{NH}_{4}{ }^{+}-\mathrm{N}$ has no significant correlation with AOA amoA or AOB amoA gene, which was similar to the study of rhizosphere sediments of riparian zone (Wang et al. 2019c). This was likely due to the relatively high $\mathrm{NH}_{4}{ }^{+}-\mathrm{N}$ concentration in lake sediments $\left(19.12 \sim 44.13 \mathrm{mg} \mathrm{kg}^{-1}\right)$ in the present study, compared to that in soils $\left(7 \sim 20 \mathrm{mg} \mathrm{kg}^{-1}\right)$ (Shi et al. 2018). Such high $\mathrm{NH}_{4}{ }^{+}-\mathrm{N}$ concentrations can supply sufficient reaction substrates, rather than being a limiting factor, for aerobic ammonia oxidizing bacteria. 
The half-saturation constants $\left(K_{\mathrm{m}}\right)$ of $C a$. $N$. inopinata was determined to be $49 \mathrm{nM}$ by pure culture method (Daims et al. 2017; Kits et al. 2017). And the AOA $K_{\mathrm{m}}$ was $3.6 \mathrm{nM} \sim 4 \mu \mathrm{M}$ (Kits et al. 2017). Compared with comammox Nitrospira and AOA, AOB has higher half-saturation constants and lower substrate affinity. Its $K_{\mathrm{m}}\left(\mathrm{NH}_{3}\right)$ ranged from 6 to $11 \mu \mathrm{M}$ (Lehtovirta-Morley 2019; Prosser and Nicol 2012). At present, there is no clear conclusion on the rate of ammonia oxidation of comammox bacteria. However, when the total ammonia nitrogen concentration of $\mathrm{Ca}$. $N$. inopinata was as low as 5 $\mu \mathrm{mol} \mathrm{L}{ }^{-1}$, the maximum oxidation rate of total ammonium $\left(V_{\max }\right)$ can reach $14.8 \mu \mathrm{mol} \mathrm{N}(\mathrm{mg} \bullet \mathrm{protein} \bullet \mathrm{h})$ ${ }^{-1}$ (Daims et al. 2017; Kits et al. 2017). In future studies, more attention should be paid to the ecological role of comammox in different habitats, especially in low ammonia nitrogen environment, and its contribution to nitrification rate in the nitrogen cycle.

In this study, three genus of comammox bacteria were found in an urban eutrophic lake sediments, but they were all classified as comammox clade A. The average abundance of the comammox bacteria was $2.43 \times 10^{8}$ copies $\mathrm{g}^{-1}$ dry sediment in the urban Lake Donghu. However, the comammox bacteria abundance in the five sub-lakes showed differences due to various water quality conditions. Interestingly, better water quality favored the growth of comammox bacteria. In addition, the abundance of the comammox amoA gene in each sub-lake was higher than that of the AOA amoA, and AOB $a m o A$ gene. The abundance of comammox amoA gene was positively correlated with AOA amoA, and AOB amoA gene abundance, but weakly correlated with anammox 16S rRNA gene. Comammox also had a positive correlation with sediment $\mathrm{OM}$ and $\mathrm{TN}$, but negatively correlated with water column $\mathrm{NO}_{3}{ }^{-}-\mathrm{N}, \mathrm{TP}$ and $\mathrm{NH}_{4}{ }^{+}-\mathrm{N}$. These findings suggested that comammox should play an important ecological role in the nitrogen cycle of urban lake sediments.

\section{Acknowledgments}


This work was supported by the National Natural Science Foundation of China (41371452, U1802241, 91647208).

\section{Conflict of Interest}

The authors state that they have no conflict of interest to declare.

\section{References}

Annavajhala, M.K., Kapoor, V., Santo-Domingo, J. and Chandran, K. (2018) Comammox functionality identified in diverse engineered biological wastewater treatment systems. Environ Sci Technol Lett 5, 110-116.

Bao, S.D. (1981) Soil agricultural chemical analysis. Beijing: China Agricultural Press.

Bartelme, R.P., McLellan, S.L. and Newton, R.J. (2017) Freshwater recirculating aquaculture system operations drive biofilter bacterial community shifts around a stable nitrifying consortium of ammonia-oxidizing archaea and comammox Nitrospira. Front Microbiol 8, 101.

Camejo, P.Y., Domingo, J.S., McMahon, K.D. and Noguera, D.R. (2017) Genome-Enabled insights into the ecophysiology of the comammox bacterium "Candidatus Nitrospira nitrosa". Msystems 2, e00059-17.

Chen, G.Y., Qiu, S.L. and Zhou, Y.Y. (2009) Diversity and abundance of ammonia-oxidizing bacteria in eutrophic and oligotrophic basins of a shallow Chinese lake (Lake Donghu). Res Microbiol 160, $173-178$.

Chen, J. and Gu, J.D. (2017) Faunal burrows alter the diversity, abundance, and structure of AOA, AOB, anammox and n-damo communities in coastal mangrove sediments. Microb Ecol 74, $140-156$.

Costa, E., Perez, J. and Kreft, J.U. (2006) Why is metabolic labour divided in nitrification? Trends 
Microbiol 14, 213-219.

Daims, H., Lebedeva, E.V., Pjevac, P., Han, P., Herbold, C., Albertsen, M. et al. (2015) Complete nitrification by Nitrospira bacteria. Nature 528, 504-509.

Dang, H.Y., Li, J., Chen, R.P., Wang, L., Guo, L.Z., Zhang, Z.N. and Klotz, M.G. (2010) Diversity, abundance, and spatial distribution of sediment ammonia-oxidizing betaproteobacteria in response to environmental gradients and coastal eutrophication in Jiaozhou Bay, China. Appl Environ Microbiol 76, 4691-4702.

Fowler, S.J., Palomo, A., Dechesne, A., Mines, P.D. and Smets, B.F. (2018) Comammox Nitrospira are abundant ammonia oxidizers in diverse groundwater-fed rapid sand filter communities. Environ Microbiol 20, 1002-1015.

Gao, J.F., Fan, X.Y., Pan, K.L., Li, H.Y. and Sun, L.X. (2016) Diversity, abundance and activity of ammonia-oxidizing microorganisms in fine particulate matter. Sci Rep 6, 38785.

Herrmann, M., Scheibe, A., Avrahami, S. and Kusel, K. (2011) Ammonium availability affects the ratio of ammonia-oxidizing bacteria to ammonia-oxidizing archaea in simulated creek ecosystems. Appl Environ Microbiol 77, 1896-1899.

Hou, J., Song, C.L., Cao, X.Y. and Zhou, Y.Y. (2013) Shifts between ammonia-oxidizing bacteria and archaea in relation to nitrification potential across trophic gradients in two large Chinese lakes (Lake Taihu and Lake Chaohu). Water Res 47, 2285-2296.

Hu, H.W. and He, J.Z. (2017) Comammox-a newly discovered nitrification process in the terrestrial nitrogen cycle. J Soils Sediments 17, 2709-2717.

Hulsen, T., de Vlieg, J. and Alkema, W. (2008) BioVenn-a web application for the comparison and visualization of biological lists using area-proportional Venn diagrams. BMC Genom 9, 488. 
Ji, B., Liang, J.C., Ma, Y.Q., Zhu, L. and Liu, Y. (2019) Bacterial community and eutrophic index analysis of the East Lake. Environ Pollut 252, 682-688.

Ji, G.D., Wang, R.J., Zhi, W., Liu, X.X., Kong, Y.P. and Tan, Y.F. (2012) Distribution patterns of denitrification functional genes and microbial floras in multimedia constructed wetlands. Ecol Eng 44, 179-188.

Jiang, Q., Xia, F., Zhu, T., Wang, D. and Quan, Z. (2019) Distribution of comammox and canonical ammonia oxidizing bacteria in tidal flat sediments of the Yangtze River estuary at different depths over four seasons. J Appl Microbiol 127, 533-543.

Kits, K.D., Sedlacek, C.J., Lebedeva, E.V., Han, P., Bulaev, A., Pjevac, P. et al. (2017) Kinetic analysis of a complete nitrifier reveals an oligotrophic lifestyle. Nature 549, 269-272.

Konneke, M., Bernhard, A.E., de la Torre, J.R., Walker, C.B., Waterbury, J.B. and Stahl, D.A. (2005) Isolation of an autotrophic ammonia-oxidizing marine archaeon. Nature 437, 543-546.

Kuypers, M.M.M., Sliekers, A.O., Lavik, G., Schmid, M., Jorgensen, B.B., Kuenen, J.G. et al. (2003) Anaerobic ammonium oxidation by anammox bacteria in the Black Sea. Nature 422, 608-611.

Lehtovirta-Morley, L.E. (2019) Ammonia oxidation: Ecology, physiology, biochemistry and why they must all come together. FEMS Microbiol Lett 365, 9.

Lepš, J. and Šmilauer, P. (2003) Multivariate analysis of ecological data using CANOCO. New York: Cambridge University Press.

Meinhardt, K.A., Bertagnolli, A., Pannu, M.W., Strand, S.E., Brown, S.L. and Stahl, D.A. (2015) Evaluation of revised polymerase chain reaction primers for more inclusive quantification of ammonia-oxidizing archaea and bacteria. Environ Microbiol Rep 7, 354-363.

Mulder, A., Vandegraaf, A.A., Robertson, L.A. and Kuenen, J.G. (1995) Anaerobic ammonium 
oxidation discovered in a denitrifying fluidized-red reactor. FEMS Microbiol Ecol 16, 177-183.

Palomo, A., Fowler, S.J., Gulay, A., Rasmussen, S., Sicheritz-Ponten, T. and Smets, B.F. (2016)

Metagenomic analysis of rapid gravity sand filter microbial communities suggests novel physiology of Nitrospira spp. ISME J 10, 2569-2581.

Pinto, A.J., Marcus, D.N., Ijaz, U.Z., Bautista-de Lose Santos, Q.M., Dick, G.J. and Raskin, L. (2016) Metagenomic evidence for the presence of comammox Nitrospira-like bacteria in a drinking water system. Msphere 1.

Pjevac, P., Schauberger, C., Poghosyan, L., Herbold, C.W., van Kessel, M.A.H.J., Daebeler, A. et al. (2017) AmoA-targeted polymerase chain reaction primers for the specific detection and quantification of comammox Nitrospira in the environment. Front Microbiol 8, 1508.

Pollet, T., Berdjeb, L., Chardon, C. and Jacquet, S. (2018) Contrasting temporal patterns in ammonia-oxidizing archaeal community dynamics in two peri-alpine lakes with different trophic status. Aquat Microbiol Ecol 81, 95-108.

Prosser, J.I. and Nicol, G.W. (2012) Archaeal and bacterial ammonia-oxidisers in soil: the quest for niche specialisation and differentiation. Trends Microbiol 20, 523-531.

Roots, P., Wang, Y.B., Rosenthal, A.F., Griffin, J.S., Sabba, F., Petrovich, M. et al. (2019) Comammox Nitrospira are the dominant ammonia oxidizers in a mainstream low dissolved oxygen nitrification reactor. Water Res $\mathbf{1 5 7 , 3 9 6 - 4 0 5 . ~}$

Schloss, P.D., Westcott, S.L., Ryabin, T., Hall, J.R., Hartmann, M., Hollister, E.B. et al. (2009) Introducing mothur: open-source, platform-independent, community-supported software for describing and comparing microbial communities. Appl Environ Microbiol 75, 7537-7541.

Shi, X.Z., Hu, H.W., Wang, J.Q., He, J.Z., Zheng, C.Y., Wan, X.H. and Huang, Z.Q. (2018) Niche 
separation of comammox Nitrospira and canonical ammonia oxidizers in an acidic subtropical forest soil under long-term nitrogen deposition. Soil Biol Biochem 126, 114-122.

Shiozaki, T., Ijichi, M., Isobe, K., Hashihama, F., Nakamura, K., Ehama, M. et al. (2016) Nitrification and its influence on biogeochemical cycles from the equatorial pacific to the Arctic Ocean. ISME J 10, 2184-2197.

Tamura, K., Stecher, G., Peterson, D., Filipski, A. and Kumar, S. (2013) MEGA6: Molecular Evolutionary Genetics Analysis version 6.0. Mol Biol Evol 30, 2725-2729.

van Kessel, M.A.H.J., Speth, D.R., Albertsen, M., Nielsen, P.H., Op den Camp, H.J.M., Kartal, B. et al. (2015) Complete nitrification by a single microorganism. Nature 528, 555-559.

Wang, J.C., Wang, J.L., Rhodes, G., He, J.Z. and Ge, Y. (2019a) Adaptive responses of comammox Nitrospira and canonical ammonia oxidizers to long-term fertilizations: Implications for the relative contributions of different ammonia oxidizers to soil nitrogen cycling. Sci Total Environ 668 , 224-233.

Wang, M.Y., Huang, G.H., Zhao, Z.R., Dang, C.Y., Liu, W. and Zheng, M.S. (2018) Newly designed primer pair revealed dominant and diverse comammox amoA gene in full-scale wastewater treatment plants. Bioresour Technol 270, 580-587.

Wang, W.D., Liu, W.Y., Wu, D., Wang, X.X. and Zhu, G.B. (2019b) Differentiation of nitrogen and microbial community in the littoral and limnetic sediments of a large shallow eutrophic lake (Chaohu Lake, China). J Soils Sediments 19, 1005-1016.

Wang, X.M., Wang, S.Y., Shi, G.S., Wang, W.D. and Zhu, G.B. (2019c) Factors driving the distribution and role of AOA and AOB in Phragmites communis rhizosphere in riparian zone. J Basic Microbiol 59, 425-436. 
Wang, Y.L., Ma, L.P., Mao, Y.P., Jiang, X.T., Xia, Y., Yu, K. et al. (2017) Comammox in drinking water systems. Water Res 116, 332-341.

Wu, L.N., Shen, M.Y., Li, J., Huang, S., Li, Z., Yan, Z.B. and Peng, Y.Z. (2019) Cooperation between partial-nitrification, complete ammonia oxidation (comammox), and anaerobic ammonia oxidation (anammox) in sludge digestion liquid for nitrogen removal. Environ Pollut 254.

Wu, Y., Xiang, Y., Wang, J., Zhong, J., He, J. and Wu, Q.L. (2010) Heterogeneity of archaeal and bacterial ammonia-oxidizing communities in Lake Taihu, China. Environ Microbiol Rep 2, $569-576$.

Xia, F., Wang, J.G., Zhu, T., Zou, B., Rhee, S.K. and Quan, Z.X. (2018) Ubiquity and diversity of complete ammonia oxidizers (comammox). Appl Environ Microbiol 84, e01390-18.

Yang, Y.Y., Li, N.N., Zhao, Q., Yang, M.X., Wu, Z., Xie, S.G. and Liu, Y. (2016a) Ammonia-oxidizing archaea and bacteria in water columns and sediments of a highly eutrophic plateau freshwater lake. Environ Sci Pollut R 23, 15358-15369.

Yang, Y.Y., Zhang, J.X., Zhao, Q., Zhou, Q.H., Li, N.N., Wang, Y.L. et al. (2016b) Sediment ammonia-oxidizing microorganisms in two plateau freshwater lakes at different trophic states. Microb Ecol 71, 257-265.

Yu, C.D., Hou, L.J., Zheng, Y.L., Liu, M., Yin, G.Y., Gao, J. et al. (2018) Evidence for complete nitrification in enrichment culture of tidal sediments and diversity analysis of clade a comammox Nitrospira in natural environments. Appl Microbiol Biotechnol 102, 9363-9377.

Zhao, J.W., Xu, Y.F., Peng, L., Liu, G.L., Wan, X.Q., Hua, Y.M. et al. (2019a) Diversity of anammox bacteria and abundance of functional genes for nitrogen cycling in the rhizosphere of submerged macrophytes in a freshwater lake in summer. J Soils Sediments 19, 3648-3656. 
Zhao, Z.R., Huang, G.H., He, S.S., Zhou, N., Wang, M.Y., Dang, C.Y. et al. (2019b) Abundance and community composition of comammox bacteria in different ecosystems by a universal primer set. Sci Total Environ 691, 146-155.

Zheng, M.S., Wang, M.Y., Zhao, Z.R., Zhou, N., He, S.S., Liu, S.F. et al. (2019) Transcriptional activity and diversity of comammox bacteria as a previously overlooked ammonia oxidizing prokaryote in full-scale wastewater treatment plants. Sci Total Environ 656, 717-722.

\section{Table Legends}

Table 1. Physicochemical characteristics (mean \pm SD.) of the overlying water and sediments in the five sampling sub-lakes.

Table S1. Quantitative PCR primers used in this study. 


\section{Figure Legends}

Fig. 1 Sampling sites in Lake Donghu.

Fig. 2 Phylogenetic tree of representative comammox amo $A$ sequences in the lake sediment and reference sequences from Genbank. The software MEGA 6.0 was applied to construct the phylogenetic tree using the neighbor-joining method. In the 1000 re-samplings, the bootstrap value appear on the branch nodes of the evolution distance tree in different color.

Fig. 3 Abundances of four functional genes in the five sub-lakes of Lake Donghu. Error bars represented the standard deviations of the independent triplicate qPCR reactions. Different letters for the same gene indicate the significant difference amongst five sub-lakes at $P<0.05$ (Tukey's test).

Fig. 4 RDA diagram of the relationship between four functional genes with environmental factors in all samples. LGZ, Lake Guozheng; LT, Lake Tuan; LTL, Lake Tangling; LH, Lake Hou; LM, Lake Miao; S, Sedimentary environmental factor; W, Overlying water environment factor. 
Table 1. Physicochemical characteristics (mean $\pm \mathrm{SD}$ ) of the overlying water and sediments in the five sampling sub-lakes.

\begin{tabular}{|c|c|c|c|c|c|c|c|c|c|c|c|}
\hline \multirow[b]{2}{*}{$\begin{array}{c}\text { Sub-lake } \\
\text { ID }\end{array}$} & \multicolumn{4}{|c|}{ Overlying water } & \multicolumn{7}{|c|}{ Sediments } \\
\hline & $\begin{array}{c}\mathrm{DO} \\
\left(\mathrm{mg} \mathrm{L}^{-1}\right)\end{array}$ & $\begin{array}{c}\mathrm{TP} \\
\left(\mathrm{mg} \mathrm{L}^{-1}\right)\end{array}$ & $\begin{array}{c}\mathrm{TN} \\
\left(\mathrm{mg} \mathrm{L}^{-1}\right)\end{array}$ & $\begin{array}{c}\text { COD } \\
\left(\mathrm{mg} \mathrm{L}^{-1}\right)\end{array}$ & $\mathrm{pH}$ & $\begin{array}{c}\mathrm{NO}_{2}^{-}-\mathrm{N} \\
\left(\mathrm{mg} \mathrm{kg}^{-1} \mathrm{dw}\right)\end{array}$ & $\begin{array}{c}\mathrm{NO}_{3}^{-}-\mathrm{N} \\
\left(\mathrm{mg} \mathrm{kg}^{-1} \mathrm{dw}\right)\end{array}$ & $\begin{array}{c}\mathrm{NH}_{4}{ }^{+}-\mathrm{N} \\
\left(\mathrm{mg} \mathrm{kg}^{-1} \mathrm{dw}\right)\end{array}$ & $\begin{array}{c}\mathrm{OM} \\
\left(\mathrm{g} \mathrm{kg}^{-1} \mathrm{dw}\right)\end{array}$ & $\begin{array}{c}\mathrm{TN} \\
\left(\mathrm{g} \mathrm{kg}^{-1} \mathrm{dw}\right)\end{array}$ & $\begin{array}{c}\mathrm{TP} \\
\left(\mathrm{g} \mathrm{kg}^{-1} \mathrm{dw}\right)\end{array}$ \\
\hline LGZ & $9.4 \pm 0.1 \mathrm{~b}$ & $0.094 \pm 0.01 \mathrm{c}$ & $0.65 \pm 0.06 \mathrm{~b}$ & $18.04 \pm 0.39 \mathrm{~d}$ & $7.53 \pm 0.01 \mathrm{~b}$ & $0.15 \pm 0.01 \mathrm{ab}$ & $13.11 \pm 0.94 b$ & $27.89 \pm 1.61 b c$ & $89.71 \pm 0.57 \mathrm{a}$ & $4.18 \pm 0.03 \mathrm{a}$ & $1.25 \pm 0.01 \mathrm{~b}$ \\
\hline LT & $8.2 \pm 0.01 \mathrm{e}$ & $0.049 \pm 0.01 \mathrm{~d}$ & $0.39 \pm 0.05 \mathrm{~b}$ & $16.27 \pm 1.15 \mathrm{~d}$ & $7.40 \pm 0.10 \mathrm{~b}$ & $0.13 \pm 0.02 \mathrm{~b}$ & $12.84 \pm 1.84 \mathrm{~b}$ & $24.89 \pm 0.42 b c$ & $78.59 \pm 0.92 \mathrm{ab}$ & $3.47 \pm 0.13 \mathrm{ab}$ & $0.61 \pm 0.01 \mathrm{~d}$ \\
\hline LTL & $8.5 \pm 0.01 \mathrm{~d}$ & $0.12 \pm 0.01 b c$ & $1.15 \pm 0.04 \mathrm{~b}$ & $24.71 \pm 1.45 \mathrm{c}$ & $8.01 \pm 0.05 \mathrm{a}$ & $0.15 \pm 0.02 \mathrm{ab}$ & $13.10 \pm 0.48 b$ & $19.12 \pm 1.07 \mathrm{c}$ & $70.10 \pm 2.71 b$ & $2.89 \pm 0.05 \mathrm{~b}$ & $0.77 \pm 0.01 \mathrm{c}$ \\
\hline LH & $8.7 \pm 0.03 c$ & $0.13 \pm 0.01 \mathrm{~b}$ & $0.91 \pm 0.03 b$ & $28.98 \pm 0.78 b$ & $8.03 \pm 0.03 \mathrm{a}$ & $0.21 \pm 0.01 \mathrm{a}$ & $16.73 \pm 1.95 b$ & $35.63 \pm 5.13 b$ & $56.26 \pm 0.28 \mathrm{c}$ & $2.81 \pm 0.04 \mathrm{~b}$ & $0.82 \pm 0.02 \mathrm{a}$ \\
\hline LM & $10.9 \pm 0.05 \mathrm{a}$ & $0.23 \pm 0.02 \mathrm{a}$ & $2.36 \pm 0.09 \mathrm{a}$ & $36.48 \pm 1.64 \mathrm{a}$ & $7.88 \pm 0.01 \mathrm{a}$ & $0.16 \pm 0.01 \mathrm{ab}$ & $28.10 \pm 4.90 \mathrm{a}$ & $44.13 \pm 6.20 \mathrm{a}$ & $72.82 \pm 8.90 \mathrm{~b}$ & $3.52 \pm 0.57 \mathrm{ab}$ & $2.82 \pm 0.01 \mathrm{c}$ \\
\hline
\end{tabular}

LGZ: Lake Guozheng. LT: Lake Tuan. LTL: Lake Tangling. LH: Lake Hou. LM: Lake Miao.

SD: standard deviation, dw: dry weight.

Different letters indicate significant differences amongst sub-lakes $(P<0.05)$.

Table S1. Quantitative PCR primers used in this study

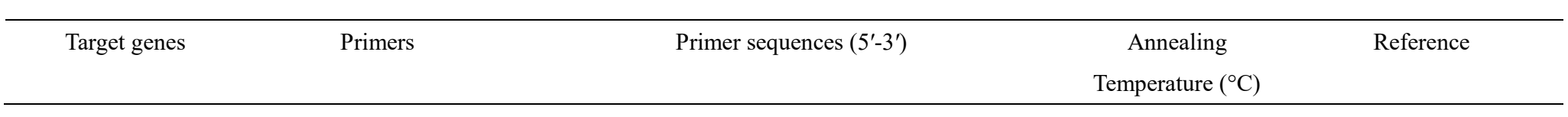




\begin{tabular}{|c|c|c|c|c|}
\hline \multirow[t]{2}{*}{ Comammox amoA } & Ntsp-amoA $162 \mathrm{~F}$ & GGATTTCTGGNTSGATTGGA & 52 & (Fowler et al. 2018) \\
\hline & Ntsp-amoA 359R & WAGTTNGACCACCASTACCA & & \\
\hline \multirow[t]{2}{*}{ AOA $а т о A$} & GenAOAF & ATAGAGCCTCAAGTAGGAAAGTTCTA & 53 & (Meinhardt et al. 2015) \\
\hline & GenAOAR & CCAAGCGGCCATCCAGCTGTATGTCC & & \\
\hline \multirow[t]{2}{*}{ AOB атоA } & amoA-1Fmod & CTGGGGTTTCTACTGGTGGTC & 52 & (Meinhardt et al. 2015) \\
\hline & GenAOBR & GCAGTGATCATCCAGTTGCG & & \\
\hline \multirow[t]{2}{*}{ Anammox 16S rRNA } & AMX809F & GCCGTA AACGATGGGCACT & 53 & (Ji et al. 2012) \\
\hline & AMX1066R & AACGTCTCACGACACGAGCTG & & \\
\hline
\end{tabular}




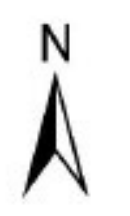

Legend:

- Sampling sites

\begin{tabular}{lllll}
0 & 1.5 & 3 & 4.5 & 6 \\
\hline
\end{tabular}

Water

Road

Bridge 


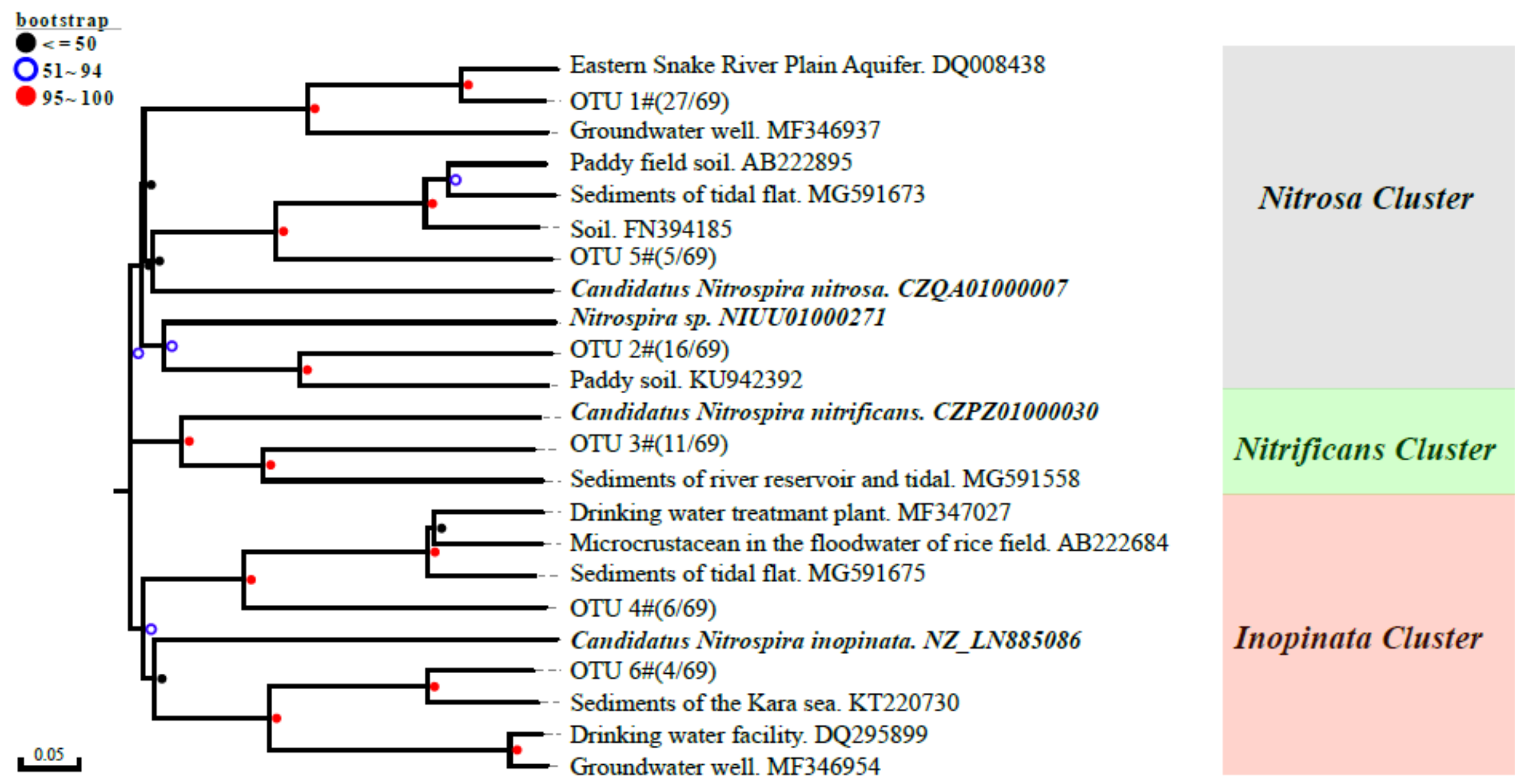




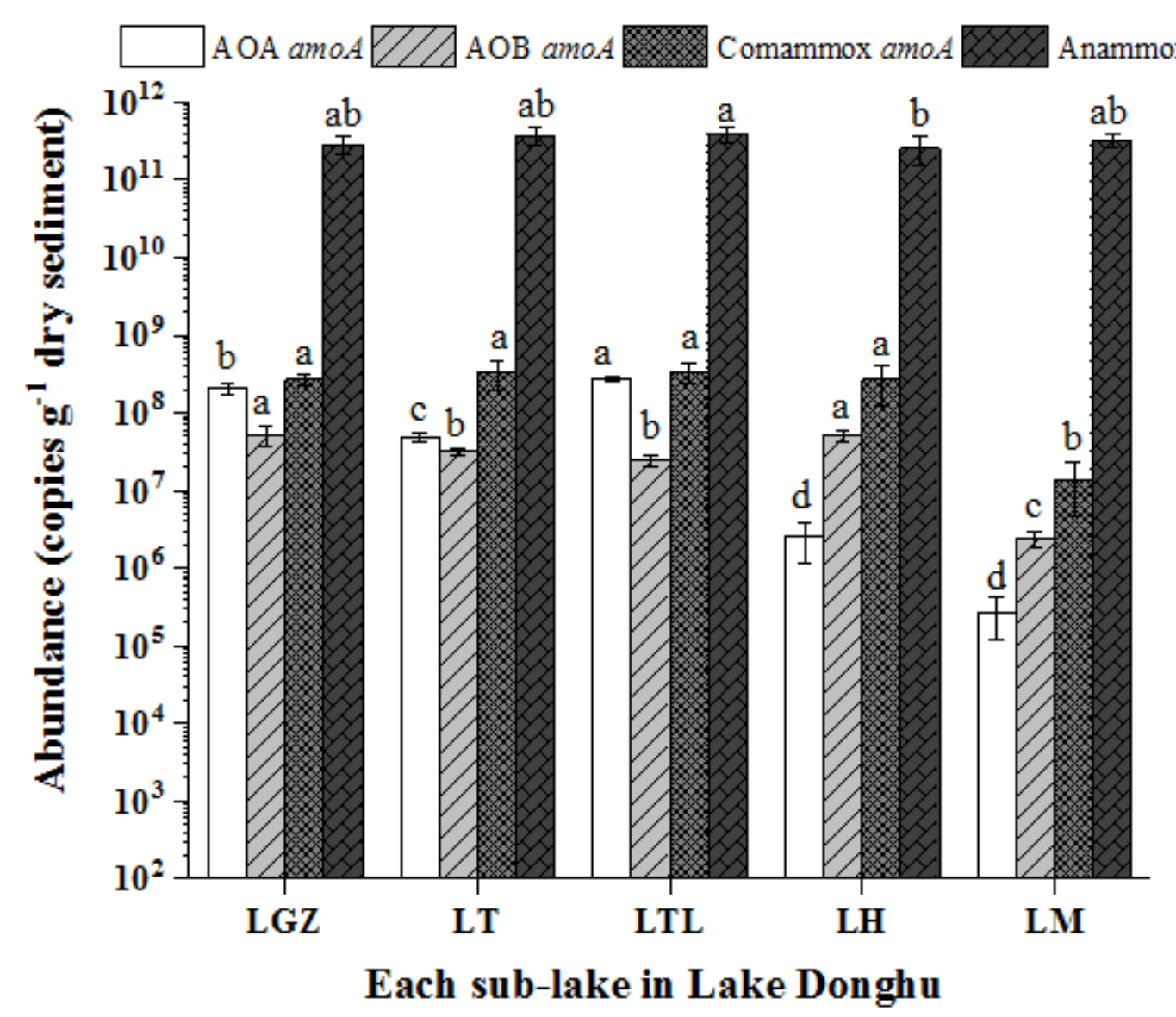




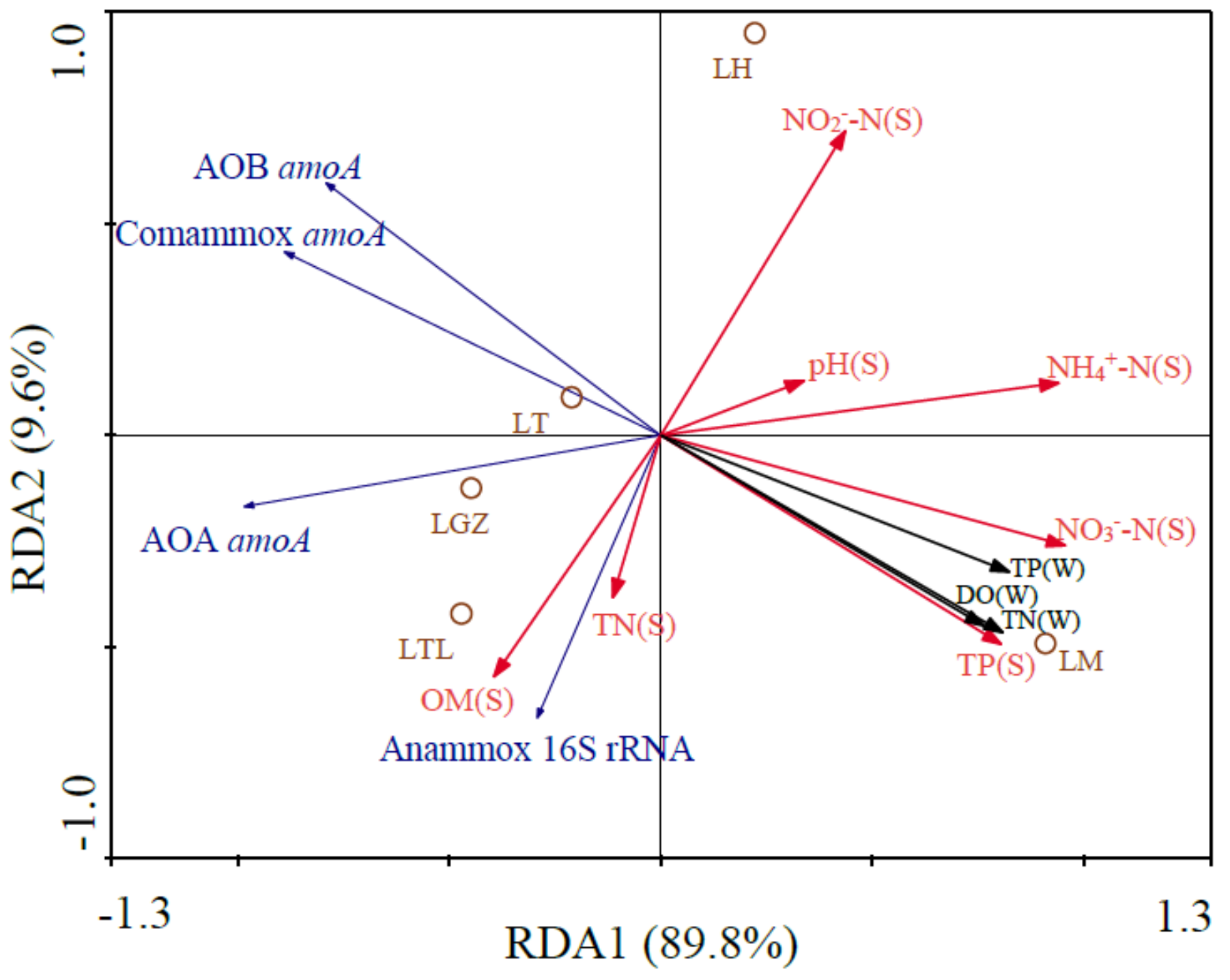

